\title{
Smoking cessation affects human platelet activation induced by collagen
}

\author{
YUKO KITO $^{1,2}$, MAMI IIDA ${ }^{3}$, KUMIKO TANABE $^{1}$, TAKASHI ONUMA $^{1,2}$, MASANORI TSUJIMOTO $^{4}$, \\ KIYOSHI NAGASE ${ }^{1}$, HARUHIKO TOKUDA ${ }^{5}$, TORU IWAMA ${ }^{4}$, OSAMU KOZAWA ${ }^{2}$ and HIROKI IIDA ${ }^{1}$
}

\author{
Departments of ${ }^{1}$ Anesthesiology and Pain Medicine, and ${ }^{2}$ Pharmacology, Gifu University Graduate School of Medicine, \\ Gifu 501-1194; ${ }^{3}$ Gifu Prefectural General Medical Center, Gifu 500-8717; ${ }^{4}$ Department of Neurosurgery, \\ Gifu University Graduate School of Medicine, Gifu 501-1194; ${ }^{5}$ Department of Clinical Laboratory, \\ National Center for Geriatrics and Gerontology, Obu, Aichi 485-8520, Japan
}

Received February 3, 2019; Accepted July 30, 2019

DOI: $10.3892 /$ etm.2019.8025

\begin{abstract}
It is firmly established that smoking is a risk factor of cardiovascular disease, stroke and peripheral vascular disease. Although smoking alters the hemostatic process, the influence of smoking on human platelet activation remains controversial. For patients undergoing surgery, cessation of smoking prior to the procedure is recommended as it increases the risk of postoperative morbidity or mortality. The presented study investigated the effects of smoking cessation on human platelet activation induced via collagen ( $\mathrm{n}=19$ patients). Blood samples were taken on four occasions: Before smoking cessation, and at 4,8 and 12 weeks after smoking cessation. Platelet aggregation using citrated platelet-rich plasma (PRP) was monitored using a PA-200 aggregometer, which determined the size of platelet aggregates using laser scattering methods. A low dose of collagen $(1 \mu \mathrm{g} / \mathrm{ml})$ accelerated platelet aggregation at 4 or 8 weeks after smoking cessation when compared with results before cessation. After 12 weeks, levels of platelet aggregation induced by collagen were almost equal to those recorded prior to smoking cessation. The secretion levels of collagen-induced platelet-derived growth factor (PDGF)-AB at 4 or 8 weeks after smoking cessation were significantly higher than those before smoking was stopped. Furthermore, smoking cessation markedly strengthened the collagen-induced phosphorylation of p38 mitogen-activated protein (MAP) kinase after 4 weeks. The results of the current study indicated that smoking cessation causes temporary short-term human platelet hyper-activation. The further suggest that the incidence of complications due to human platelet hyper-reactivity may be lowered by considering the period of smoking abstinence.
\end{abstract}

Correspondence to: Professor Hiroki Iida, Department of Anesthesiology and Pain Medicine, Gifu University Graduate School of Medicine, 1-1 Yanagido Street, Gifu 501-1194, Japan E-mail: iida@gifu-u.ac.jp

Key words: platelet, platelet activation, smoking, smoking cessation, collagen

\section{Introduction}

Smoking has been recognized to cause serious health problems. It is established that smoking is a risk factor of diseases such as myocardial infarction $(1,2)$, stroke (3) and peripheral vascular diseases (4). Smoking causes vascular thrombosis by altering the hemostatic process via changes in the function of endothelial cells and platelets affected by fibrinogen, or coagulation factors (2). Concerning the platelet function, the number of small aggregates of platelets in smokers' plasma is significantly higher than in non-smokers in the absence of chemical stimulants (5). Furthermore, acute smoking exposure in habitual smokers increases the platelet aggregation and induces greater resistance to thrombolysis than in non-smokers (5), and the mean platelet volume and platelet distribution width, which are indicators of platelet activation, are significantly higher in smokers than in non-smokers (6). However, there are no significant differences between smokers and non-smokers in platelet aggregation induced by ADP (7). Given these conflicting previous findings, the influence of smoking on platelet activation has not been fully clarified.

Quitting of smoking is recommended in patients undergoing surgery, because smoking increases the risk of postoperative morbidity or mortality $(8,9)$. Smoking cessation reduces the rate of postoperative pulmonary complications such as pneumonia (10) or wound-healing complications such as surgical site infection (9). At least eight weeks are required for the disappearance of the cough-promoting effect of smoking and reduction of postoperative pulmonary complications (11) and more than four weeks are required to reduce wound-healing complications (10). In addition, surgical patients are at risk of developing venous thrombo-embolism (12). The main cellular components of thrombo-embolism are platelets, endothelial cells, monocytes and erythrocytes (12). However, the influence of smoking cessation for surgery on platelet activation is not fully known. Platelet aggregability in long-term smokers is not reversible in four weeks (13). However, only two weeks of smoking cessation reduced the ADP or collagen-induced platelet aggregations through suppression of oxidative stress (14). 
Collagen initiates human platelet activation in the process of thrombus formation. The interactions of platelet glycoprotein VI with the collagen of the exposed vessel wall and the complex of platelet glycoprotein Ib/IX/V with von Willebrand factor (vWF) result in the adhesion of platelets to the site of injury. After this adhesion, thromboxane $\mathrm{A}_{2}$ and ADP lead to the activation of glycoprotein IIb/IIIa $(\alpha \operatorname{IIb} \beta 3)$ and thrombosis formation (15). Thrombus formation is also associated with the secretion of granule contents, such as platelet-derived growth factor (PDGF)-AB (16). In the plasma of smokers, platelet $\alpha$-granule constituents are increased, leading to platelet aggregation (2). Smoking have higher P2Y12 receptor expression on platelets, which increases the ADP-induced platelet aggregation (2). However, the effects of smoking or smoking cessation on platelet activation and the intracellular mechanism are not fully clarified. Furthermore, the time-dependent changes in the platelet function associated with smoking cessation remain unclear.

We previously demonstrated that $\operatorname{ADP}(17,18)$ and collagen $(19,20)$ induce the secretion of PDGF-AB via the activation of p38 mitogen-activated protein (MAP) kinase in human platelets. In the present study, to investigate the effects of smoking and the cessation of smoking on human platelet activation, we examined the effects of collagen on platelet aggregation, the secretion of PDGF-AB and the underlying mechanism in patients who were quitting smoking for surgery over time for 12 weeks.

\section{Materials and methods}

Materials. Collagen was purchased from Takeda Austria $\mathrm{GmbH}$ (Linz, Austria). A PDGF-AB enzyme-linked immunosorbent assay (ELISA) kit was purchased from R\&D System, Inc. (Minneapolis, MN, USA). Phospho-specific p38 MAP kinase antibodies and p38 MAP kinase antibodies were obtained from Cell Signaling, Inc. (Beverly, MA, USA). GAPDH antibodies were purchased from Santa Cruz Biotechnology (Santa Cruz, CA, USA). All other materials and chemicals were obtained from commercial sources.

Subjects. This study was approved by the Ethics Committee of Gifu University Graduate School of Medicine and Gifu Prefectural General Medical Center. All participants signed an informed consent agreement after receiving a detailed explanation of the study.

We enrolled 19 patients who visited smoking cessation outpatient services at Gifu University Graduate School of Medicine or Gifu Prefectural General Medical Center between January 2012 and November 2014. However, four patients were excluded from the current study as they only visited outpatient services once. A self-assessment was administered, and we examined the concentration of carbon monoxide (CO) exhaled to confirm smoking cessation. Blood samples were donated 4 times as follows: before smoking cessation, and 4, 8 and 12 weeks after smoking cessation. We avoided drawing blood except for at these time points in order to avoid influencing the platelet function. Blood samples were drawn from the antecubital vein by careful venipuncture in a $21-\mathrm{G}$ sterile syringe.

Human blood samples were combined with 1/10 volume of a $3.8 \%$ sodium citrate. Platelet-rich plasma (PRP) was obtained from blood samples by centrifugation at $155 \mathrm{x}$ g for $12 \mathrm{~min}$ at room temperature. Platelet-poor plasma (PPP) was prepared from the residual blood by centrifugation at 2,500 $\mathrm{x} g$ for 5 min.

Two patients with severe hypertriglycemia (No. 10 and No. 12) were excluded because their PRP and PPP were highly turbid and the samples are inappropriate for an analysis of platelet function. Therefore, a total of 13 patients who ceased smoking were analyzed in the current study.

Platelet aggregation. Platelet aggregation using citrated PRP was monitored using a PA-200 aggregometer (Kowa Co., Ltd., Tokyo, Japan), which can determine the size of platelet aggregates via particle counting using laser scattering methods (small, 9-25 $\mu \mathrm{m}$; medium, 25-50 $\mu \mathrm{m}$; large, 50-70 $\mu \mathrm{m}$ ) (21) at $37^{\circ} \mathrm{C}$ with a stirring speed of $800 \mathrm{rpm}$. Platelet aggregation was monitored for 4 min after stimulation with collagen ( $n=13$ patients). The percentage of transmittance of the isolated platelets was recorded as $0 \%$, and that of the appropriate PPP (blank) was recorded as $100 \%$.

Determination of the $E D_{50}$ value. We calculated the $\mathrm{ED}_{50}$ of collagen for the platelet aggregation using an aggregometer with the laser scattering system. The percentage of aggregation in each subject was analyzed at a dose of $0,1,3$ and $10 \mu \mathrm{g} / \mathrm{ml}$ collagen. Using the ALOKA curve software program included in the ALOKA RIA programs (ALOKA, Tokyo, Japan), a dose-response curve was plotted. From the regression equation, the collagen dose corresponding to $50 \%$ aggregation was calculated as the individual $\mathrm{ED}_{50}$ value (22).

Protein preparation after stimulation. After the stimulation with collagen, platelet aggregation was terminated by the addition of an ice-cold EDTA $(10 \mathrm{mM})$ solution. The mixture was centrifuged at $10,000 \times \mathrm{g}$ at $4^{\circ} \mathrm{C}$ for $2 \mathrm{~min}$. To measure the secreted PDGF-AB as described below, the supernatant was isolated and stored at $-30^{\circ} \mathrm{C}$ for a subsequent enzyme-linked immunosorbent assay (ELISA). For the Western blot analysis, the pellet was washed twice with phosphate-buffered saline, lysed, and immediately boiled in a lysis buffer containing $62.5 \mathrm{mM}$ Tris/Cl, $\mathrm{pH}$ 6.8, 2\% sodium dodecyl sulfate (SDS), $50 \mathrm{mM}$ dithiothreitol and $10 \%$ glycerol.

Western blotting. Western blot analysis was performed as described previously (23). Briefly, SDS-polyacrylamide gel electrophoresis (PAGE) was performed by the method described by Laemmli (24) using a $10 \%$ polyacrylamide gel. The proteins in the gel were transferred onto a polyvinylidene fluoride (PVDF) membrane, which was then blocked with $5 \%$ fat-free dry milk in Tris-buffered saline with 0.1\% Tween-20 (TBS-T, 20 mM Tris, pH 7.6, 137 mM NaCl, $0.1 \%$ Tween-20) for $2 \mathrm{~h}$ before incubation with the indicated primary antibodies. The primary antibodies used in this study were phospho-specific p38 MAP kinase antibodies, p38 MAP kinase antibodies or GAPDH antibodies. Peroxidase-labeled anti-rabbit IgG antibodies or anti-goat IgG antibodies were used as secondary antibodies. The primary and secondary antibodies were diluted to the optimum concentration with $5 \%$ fat-free dry milk in TBS-T. The peroxidase activity on the PVDF membranes was visualized on X-ray film using an 

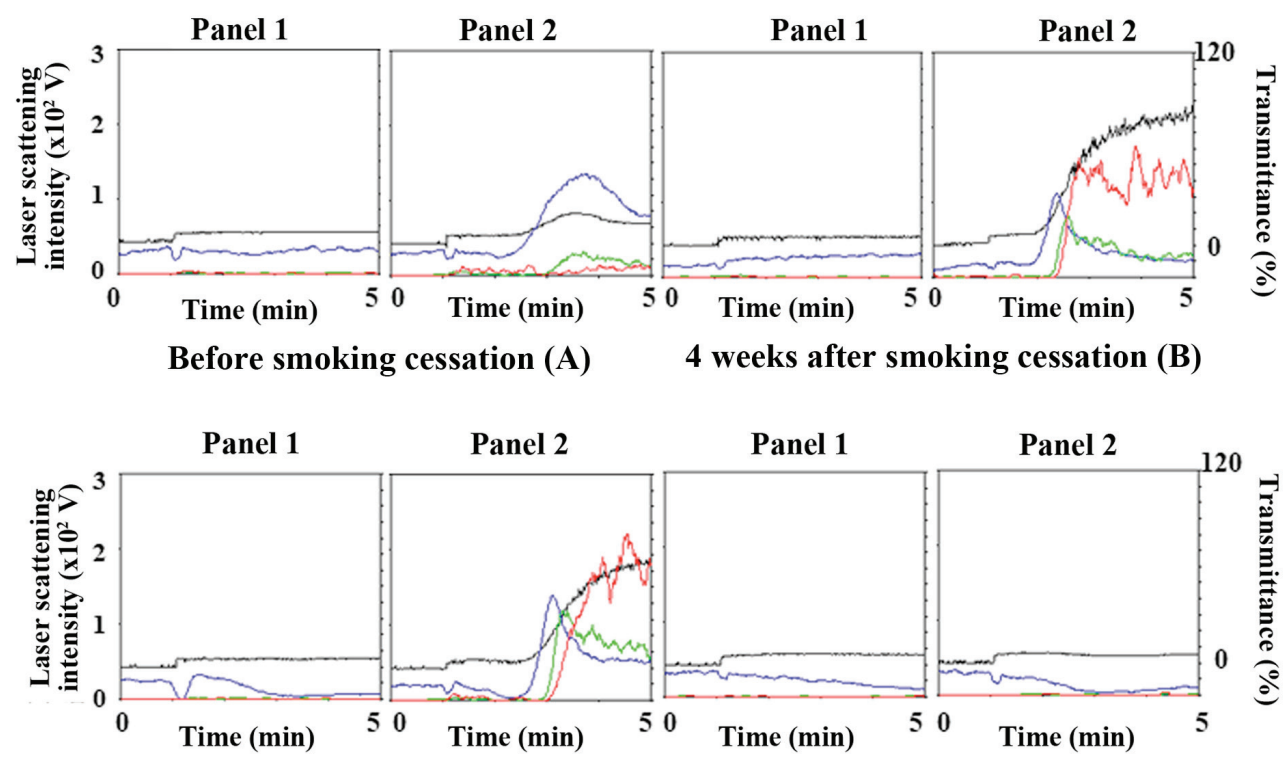

8 weeks after smoking cessation $(C) \quad 12$ weeks after smoking cessation (D)

\begin{tabular}{|c|c|c|c|c|c|c|c|c|}
\hline \multirow{2}{*}{$\begin{array}{r}\text { Panel } \\
\text { Collagen } \\
(1 \mu \mathrm{g} / \mathrm{ml})\end{array}$} & (A)-1 & (A)-2 & (B)-1 & (B)-2 & (C)-1 & (C) -2 & (D)-1 & (D)-2 \\
\hline & - & + & - & + & - & + & - & + \\
\hline \multirow{2}{*}{$\begin{array}{r}\text { Transmittance (\%) } \\
\text { Ratio (\%) }\end{array}$} & 5 & 22 & 6 & 99 & 5 & 73 & 6 & 9 \\
\hline & & & & & & & & \\
\hline small & 98 & 83 & 98 & 26 & 95 & 41 & 98 & 98 \\
\hline medium & 1 & 10 & 1 & 18 & 2 & 27 & 1 & 1 \\
\hline large & 1 & 7 & 1 & 56 & 3 & 32 & 1 & 1 \\
\hline Total & 100 & 100 & 100 & 100 & 100 & 100 & 100 & 100 \\
\hline
\end{tabular}

Figure 1. Effect of smoking cessation on platelet aggregation induced by collagen. PRP plasma was stimulated by $1 \mu \mathrm{g} / \mathrm{ml}$ of collagen or vehicle for $5 \mathrm{~min}$. Results were obtained (A) before cessation, at (B) 4 weeks, at (C) 8 weeks and at (D) 12 weeks. The reaction was terminated by adding ice-cold EDTA (10 mM) solution. (A) Representative pattern of platelet aggregation induced by collagen (high dose, $10 \mu \mathrm{g} / \mathrm{ml} ; \mathrm{low}$ dose, $1 \mu \mathrm{g} / \mathrm{ml}$ ) as detected using an aggregometer with a laser scattering system. The black curve indicates the percentage of transmittance in each sample (isolated platelets were recorded as $0 \%$; platelet-free plasma was recorded as $100 \%)$. The blue line indicates small aggregates $(9-25 \mu \mathrm{m})$ the green line indicates medium aggregates $(25-50 \mu \mathrm{m})$ and the red line indicates large aggregates $(50-70 \mu \mathrm{m})$. The distribution $(\%)$ of aggregated particle size was measured using laser scattering methods. PRP, platelet-rich plasma.

ECL Western blotting detection system as described in the manufacturer's instructions.

Measurement of PDGF-AB. The PDGF-AB levels in the samples were determined using a PDGF-AB ELISA kit in accordance with the manufacturer's instructions.

Statistical analyses. The data are presented as box-and-whisker plots representing the median \pm 25 and 75 percentile values. Statistical analyses were performed using the SPSS software program, ver. 23.0 (IBM Japan Ltd., Tokyo, Japan). The data were analyzed by Friedman test followed by Wilcoxon signed-rank test for multiple comparisons using the Bonferroni method. A P-value $<0.05$ was considered to indicate statistical significance.

\section{Results}

Effects of smoking cessation on the human platelet aggregation induced by collagen. Nineteen patients who intended to abstain from smoking participated in this study, but four who stopped visiting the hospital during the treatment period were excluded. The characteristics of the patients are detailed in Table I. The concentration of exhaled CO decreased after smoking cessation in all patients (Table I). We first examined the effect of smoking cessation on the platelet aggregation stimulated by collagen. Before smoking cessation, collagen dose-dependently elicited platelet aggregation between 1 and $10 \mu \mathrm{g} / \mathrm{ml}$. For platelet aggregation induced by a high dose of collagen $(10 \mu \mathrm{g} / \mathrm{ml})$, the transmittance of aggregation was over $100 \%$ throughout the observation periods. There were no significant differences between the findings before and after smoking cessation (data not shown). In contrast, a low dose of collagen $(1 \mu \mathrm{g} / \mathrm{ml})$ accelerated platelet aggregation at 4 or 8 weeks after smoking cessation compared with that before cessation (Fig. 1). In addition, 12 weeks after smoking cessation, the levels of platelet aggregation induced by collagen $(1 \mu \mathrm{g} / \mathrm{ml})$ had nearly returned to the levels before smoking cessation. Representative patterns of low-dose collagen $(1 \mu \mathrm{g} / \mathrm{ml})$-induced platelet aggregation are shown in Fig. 1. 


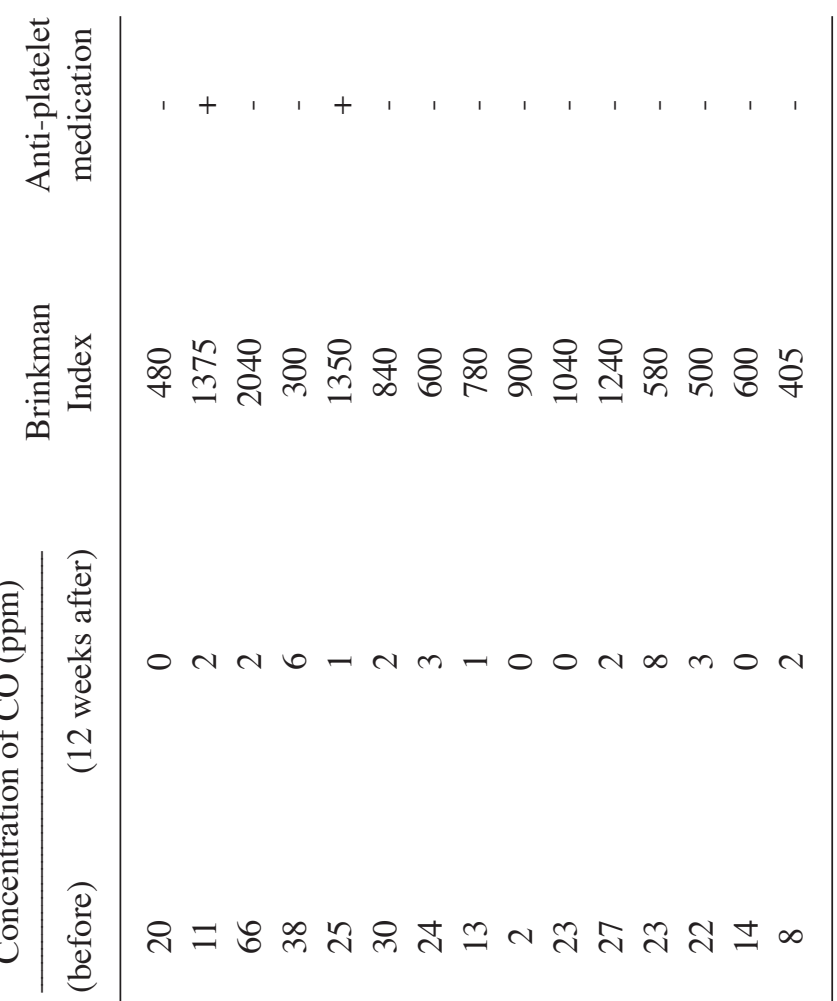



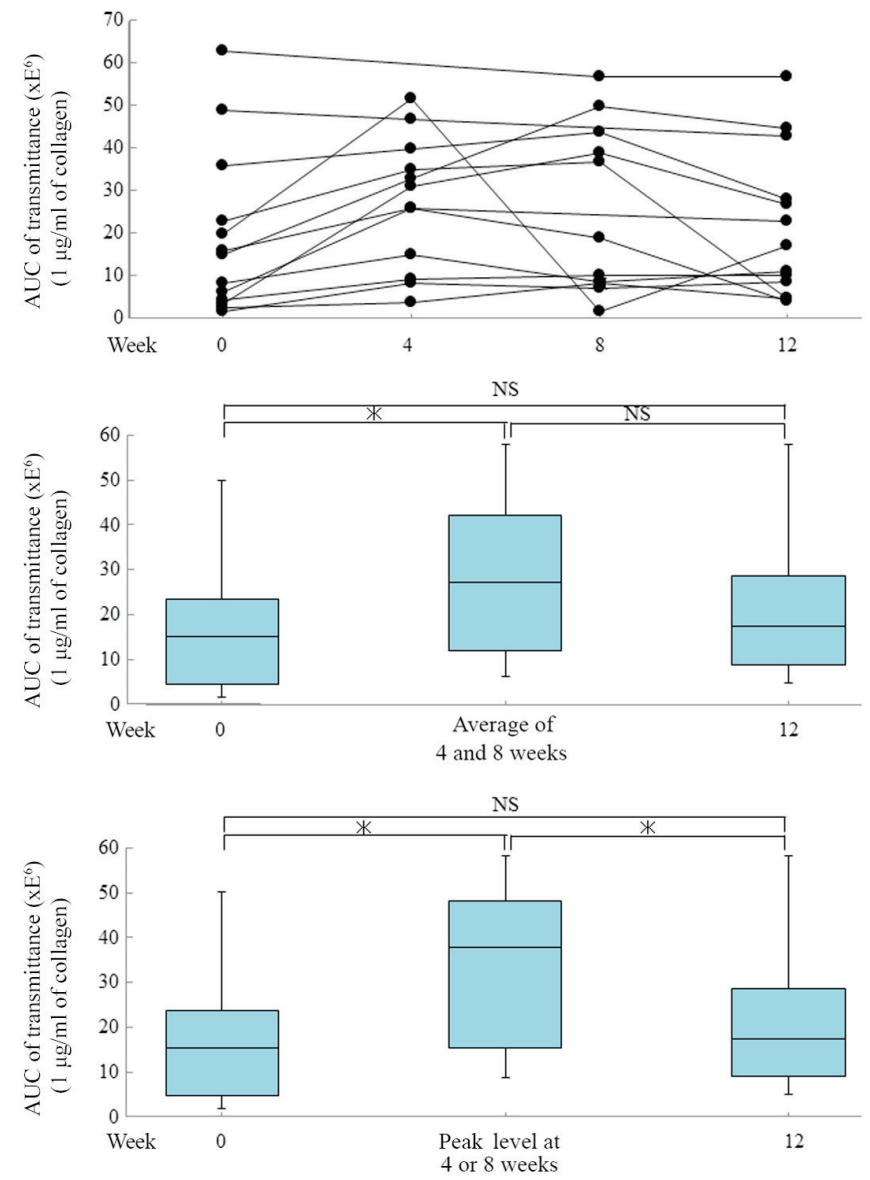

Figure 2. Effect of smoking cessation on the AUC of transmittance for platelet aggregation induced by collagen. PRP was stimulated by $1 \mu \mathrm{g} / \mathrm{ml}$ of collagen or vehicle for $5 \mathrm{~min}$. The reaction was terminated by adding ice-cold EDTA $(10 \mathrm{mM})$ solution. The values of AUC of transmittance were detected using an aggregometer with a laser scattering system ( $\mathrm{n}=13$ patients). Values before smoking cessation and at 4-8 and 12 weeks after smoking cessation in each case are presented in the upper line graph. Each upper boxplot presents the pre-smoking cessation values and the average value of 4-8 and 12 weeks after smoking cessation. Each lower boxplot presents the pre-smoking cessation value and the peak level at 4-8 and 12 weeks after smoking cessation. ${ }^{*}<0.05$ as indicated. AUC, area under the curve; PRP, platelet-rich plasma; NS, not significant.
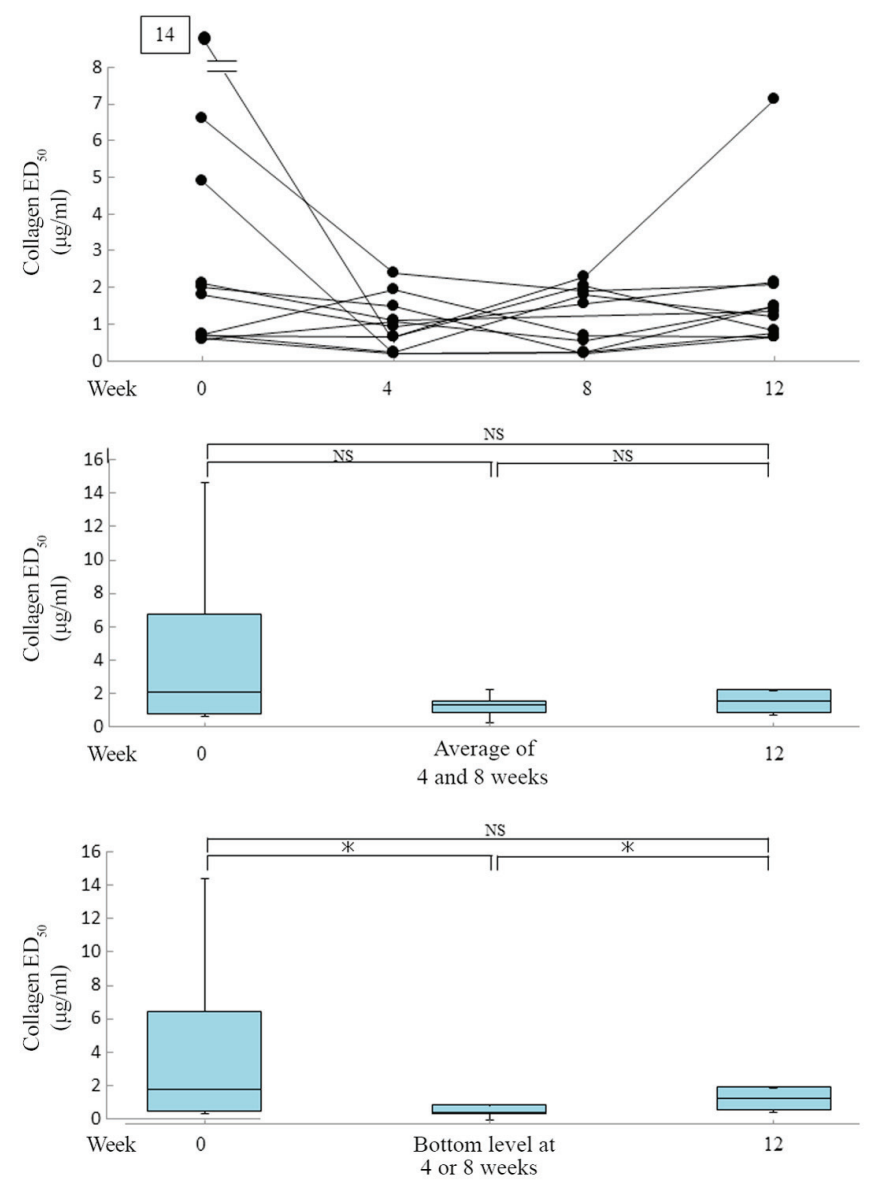

Figure 3. Effect of smoking cessation on the parameter $\left(\mathrm{ED}_{50}\right)$ for platelet aggregation induced by collagen. PRP was stimulated by $1 \mu \mathrm{g} / \mathrm{ml}$ of collagen or vehicle for $5 \mathrm{~min}$. The reaction was terminated by the addition of ice-cold EDTA $(10 \mathrm{mM})$ solution. The values of $\mathrm{ED}_{50}$ were determined using an aggregometer with a laser scattering system ( $n=13$ patients). The values obtained before smoking cessation, and at 4-8 and 12 weeks after smoking cessation in each case are presented in the upper panel. Each upper boxplot presents pre-smoking cessation values and the average values obtained at 4-8 and 12 weeks after smoking cessation. Each lower boxplot presents the pre-smoking cessation value and the $0,4-8$ and 12 weeks after smoking cessation. ${ }^{*} \mathrm{P}<0.05$ as indicated. PRP, platelet-rich plasma; NS, not significant.
Based on the analysis of the size of platelet aggregates using laser scattering methods, after 4 or 8 weeks smoking cessation, the ratio of large aggregates $(50-70 \mu \mathrm{m})$ stimulated by $1 \mu \mathrm{g} / \mathrm{ml}$ collagen was significantly increased while the ratio of small aggregates $(9-25 \mu \mathrm{m})$ was significantly decreased (data not shown). The peak value of the areas under the curve (AUCs) of transmittance stimulated by low-dose collagen $(1 \mu \mathrm{g} / \mathrm{ml})$ at 4 or 8 weeks after smoking cessation were significantly higher than the respective AUCs before smoking cessation (Fig. 2). In addition, the AUC of transmittance stimulated by $1 \mu \mathrm{g} / \mathrm{ml}$ collagen at 12 weeks after smoking cessation returned to levels similar to those before the cessation of smoking.

The bottom $\mathrm{ED}_{50}$ values of collagen $(1 \mu \mathrm{g} / \mathrm{ml})$ at 4 or 8 weeks after smoking cessation were significantly lower the respective $\mathrm{ED}_{50}$ values before smoking cessation, and at 12 weeks after the cessation, these values returned to levels similar to those observed before the cessation (Fig. 3).

Effect of smoking cessation on the collagen-induced PDGF-AB secretion in human platelets. We previously reported that collagen induces PDGF-AB secretion from human platelets (25). We further examined whether smoking cessation affects the collagen-induced PDGF-AB secretion from human platelets or not. The peak values of PDGF-AB at 4 or 8 weeks after the cessation of smoking were significantly higher than before the cessation (Fig. 4).

Considering the measured individual data, the peak or bottom value seems to be at 4 or 8 weeks. However, there is a trend but no statistically significant difference between $0,4,8$ and 12 weeks (data not shown). We'd like to clarify the tendency of short-term hyperactivation of human platelets at 4 or 8 weeks. Therefore, we presented some plots weeks 4 and 8 together (Figs. 2-4).

Effect of smoking cessation on the collagen-induced phosphorylation of 38 MAP kinase in human platelets. In our previous studies (17-20), we demonstrated that collagen-induced PDGF-AB secretion is associated with the activation of p38 MAP kinase in human platelets. Therefore, we examined 

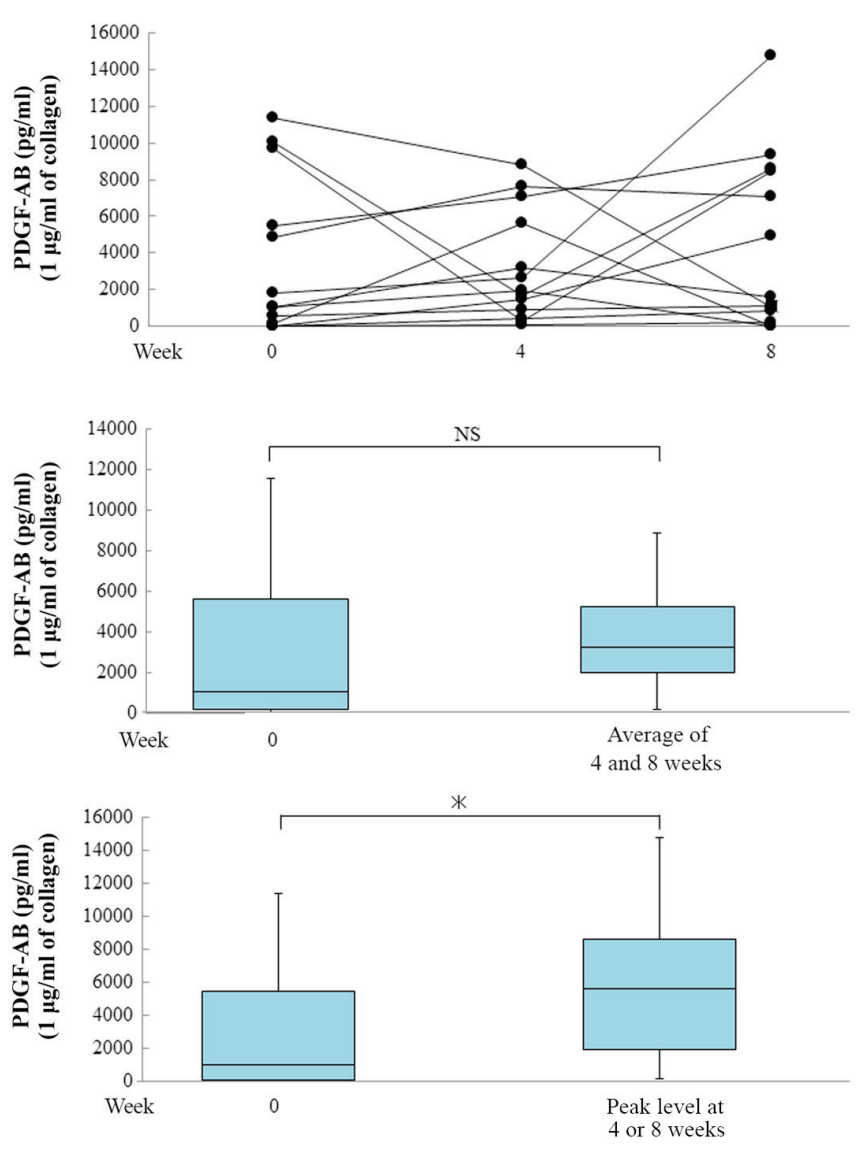

Figure 4. Effect of smoking cessation on collagen-induced PDGF-AB secretion in human platelets. PRP was stimulated by $1 \mu \mathrm{g} / \mathrm{ml}$ of collagen for $5 \mathrm{~min}$. The reaction was terminated by adding ice-cold EDTA $(10 \mathrm{mM})$ solution. The mixture was centrifuged at $10,000 \mathrm{xg}$ at $4^{\circ} \mathrm{C}$ for $2 \mathrm{~min}$ and the supernatants were subjected to ELISA. The values before smoking cessation and at 4-8 and 12 weeks after smoking cessation in each case are presented in the upper line graph. Each upper boxplot presents the pre-smoking cessation value and the average of 4-8 weeks after smoking cessation. Each lower boxplot indicates the pre-smoking cessation value and the peak level at 4-8 and 12 weeks after smoking cessation. " $\mathrm{P}<0.05$ as indicated. PDGF, platelet-derived growth factor; PRP, platelet-rich plasma; NS, not significant.

whether smoking cessation affects the collagen-induced phosphorylation of p38 MAP kinase or not. Smoking cessation markedly enhanced the levels of collagen-induced phosphorylation of p38 MAP kinase at 4 weeks compared to before smoking cessation, whereas the total p38 MAP kinase levels were not affected. Though, there is no significant difference of the levels of the collagen-induced phosphorylation of p38 MAP kinase between 4 and 8 weeks. Representative results are shown in Fig. 5.

\section{Discussion}

In the present study, we investigated the effect of smoking cessation on human platelet activation. We showed that cessation for 4 and 8 weeks induced a significantly increase in the AUC (transmittance) of collagen $(1 \mu \mathrm{g} / \mathrm{ml})$-induced platelet aggregation and a decrease in the $\mathrm{ED}_{50}$ of collagen compared with the values before smoking cessation. In addition, smoking cessation affected the distribution of aggregated particle sizes of human platelets as evaluated using laser scattering methods. These results suggest that the platelet aggregation induced by
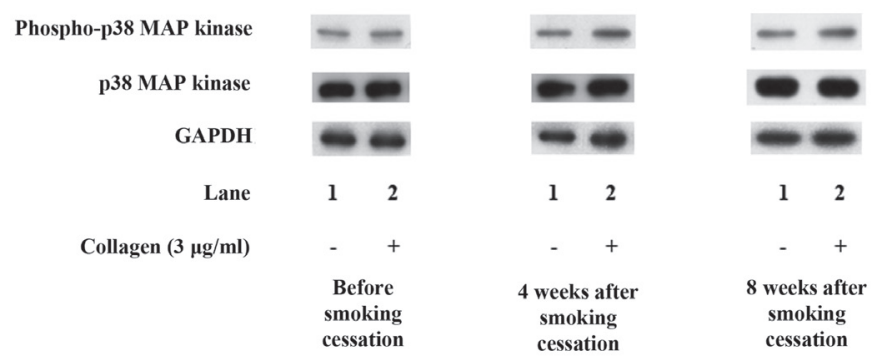

Figure 5. Effect of smoking cessation on the phosphorylation of p38 MAP kinase induced by collagen in human platelets. PRP was stimulated by $1 \mu \mathrm{g} / \mathrm{ml}$ of collagen for $5 \mathrm{~min}$. The reaction was terminated by adding ice-cold EDTA $(10 \mathrm{mM})$ solution. The lysate of platelets was harvested and subjected to SDS-PAGE using antibodies against phospho-specific p38 MAP kinase, p38 MAP kinase or GAPDH. Representative western blotting data before smoking cessation and at 4 and 8 weeks after smoking cessation are presented. MAP, mitogen activated protein; PRP, platelet-rich plasma.

low-dose collagen $(1 \mu \mathrm{g} / \mathrm{ml})$ was temporarily up-regulated by the smoking cessation. We also found that the collagen-induced PDGF-AB secretion after cessation was temporarily amplified. In our previous studies (18-20), we demonstrated that collagen or ADP induces the secretion of PDGF-AB from human platelets associated with the activation of p38 MAP kinase. From this viewpoint, we focused on the effect of smoking cessation on the p38 MAP kinase activation. We found that smoking cessation remarkably enhanced the collagen-induced phosphorylation of p38 MAP kinase. Therefore, smoking cessation may up-regulate the secretion of PDGF-AB by collagen from human platelets associated with the enhancement of p38 MAP kinase activation. These results newly suggest that smoking cessation temporary causes hyper-reactivity of human platelets, which is observed relatively short-term from 4 to 8 weeks after cessation.

Platelet activation and aggregability are more augmented in smokers than in non-smokers $(5,6,26-29)$. A previous report found that chronic exposure to cigarette smoke sustains the activation of the endothelial-coagulative system, such as by increasing vWF antigen, D-dimer, prothrombin fragment F1+2, platelet factor-4 and beta-thromboglobulin, and smoking abstinence may result in the improvement of several endothelial-coagulative system abnormalities (30).

vWF activity decreases as early as 2 months after starting smoking abstinence, and other circulating endothelial-coagulative activation markers are substantially modified at 6 and 12 months after smoking abstinence in regular smokers (30). The mean platelet volume, which is known to be a simple and convenient indicator for platelet activation, is significantly higher in regular smokers than in non-smokers $(6,28)$ and decreases significantly at three months after smoking cessation (28). In contrast, Morita et al (14) reported that only two weeks of smoking cessation in long-term smokers was able to ameliorate the enhanced platelet aggregability and intraplatelet redox imbalance. In present study, we examined the time-course changes in the platelet function including the mechanism over 12 -week at 4 -week intervals. Our present findings showing that the hyper-reactivity in human platelet was transient, occurring relatively short-term after 4 to 8 weeks of smoking cessation, is inconsistent with these previous reports. While we did not gather data at very early 
timing (e.g. 1 and 2 weeks) of the smoking cessation, smoking cessation might cause extremely complicated changes in the platelet function. At the very least, our present findings suggest that the time-dependent changes in the platelet function through the persistence of smoking cessation require physicians to monitor the temporary augmentation of the platelet aggregability.

Smoking cessation is recommended before surgery. A systematic review showed that the incidence of postoperative complications, such as general morbidity, wound complications, general infections and pulmonary or neurological complications, was significantly higher in smokers than in non-smokers (31). It has been reported that current smokers undergoing coronary artery bypass surgery have higher rates of pulmonary complications, such as postoperative pneumonia, pleural effusion and adult respiratory distress syndrome, than non-smokers, with the risk declining in patients after more than four weeks smoking cessation (32). In addition, four weeks of preoperative smoking cessation reduced the incidence of pulmonary complications after pulmonary surgery (33). Turan et al (34) demonstrated that in non-cardiac surgical patients, smoking was associated with a statistically significant increase in cardiovascular complications: In their large data set, there was a $57 \%$ increased chance of experiencing 30 -day postoperative cardiac arrest, an $80 \%$ increased chance of experiencing a myocardial infarction, and a $73 \%$ increase chance of experiencing stroke.

However, conflicting findings have also been reported; for example, a meta-analysis shows that the preoperative smoking status was not associated with cardiovascular complications (31). Surgical patients are at risk of developing thrombo-embolism during the post-operative period with and without smoking (12). However, to our knowledge, there are no clinical reports describing an increase in perioperative thrombus formation due to smoking cessation. In the present study, we found that more than 4 to 8 weeks are needed to restore the human platelet function; however, the precise duration required remains unclear. Although the incidence of cardiovascular complications tended to decrease, no statistically significant decrease $(\mathrm{P}=0.08)$ was observed after 6 to 8 weeks of smoking cessation in those who had undergone hip or knee arthroplasty (35). In addition, we could not elucidate the benefits of smoking cessation regarding the prevention of cardiovascular complications $(10,36)$. Our present findings, which show the non-linear recovery of the human platelet function according to smoking cessation, might provide a possible explanation for the phenomenon in which postoperative cardiovascular complications do not obviously decrease immediately after smoking cessation. Physicians should take measures to avoid thrombus formation in the perioperative period because smoking cessation can induce temporary hyper-activation of platelets in the short term. A longer duration of preoperative smoking cessation is likely to be desirable, and anesthesiologists should recommend the cessation of smoking as soon as possible before surgery. In addition, the incidence of complications such as mortality or pulmonary complications does not increase with short-term smoking cessation $(36,37)$, so it is not necessary to postpone an operation even if patients have only stopped smoking for a short while.
Several limitations associated with the present study warrant mention. Both the self-assessment of patients and the $\mathrm{CO}$ concentration of breathing were used for the confirmation of smoking cessation. However, the expired $\mathrm{CO}$ concentration in smokers who have refrained from smoking for $>8 \mathrm{~h}$ is almost as low as that in non-smokers. Therefore, the confirmation of complete quitting smoking during the 12-week period depended largely on the self-report of patients. Some patients had remarkable medical histories, such as hypertension, diabetes and hyperlipidemia or anti-platelet medication, which may have affected the platelet aggregation.

In conclusion, our results strongly suggest that smoking cessation causes the temporary hyper-activation of human platelets in the short term (4 to 8 weeks). Our present findings suggest that the incidence of complications due to hyper-reactivity of human platelets can be reduced by paying attention to the smoking abstinence period. We should give preoperative patients clear and strong advice to quit smoking as soon as possible and provide educational messages about the impact of smoking on surgery.

\section{Acknowledgments}

The authors would like to thank Yumiko Kurokawa (Department of Pharmacology, Gifu University Graduate School of Medicine, Gifu, Japan) for her technical assistance.

\section{Funding}

No funding was received.

\section{Availability of data and materials}

The datasets used and/or analyzed during the current study are available from the corresponding author on reasonable request.

\section{Authors' contributions}

YK and TO performed in vitro experiments and collected the data. MI, KT and TI drafted the manuscript and acquired the data. KN and MT performed statistical analysis. HT constructed the in vivo model and the associated experiments. $\mathrm{OK}$ and $\mathrm{HI}$ designed the present study, and drafted and revised the manuscript. All authors critically reviewed the manuscript and approved the final version for publication.

\section{Ethics approval and consent to participate}

The present study was approved by the Ethics Committee of Gifu University Graduate School of Medicine (approval no. 23-209) and Gifu Prefectural General Medical Center (approval no. 30) All participants signed an informed consent agreement after receiving a detailed explanation of the study protocol.

\section{Patient consent for publication}

Not applicable. 


\section{Competing interests}

The authors declare that they have no competing interests.

\section{References}

1. Ambrose JA and Barua RS: The pathophysiology of cigarette smoking and cardiovascular disease: An update. J Am Coll Cardiol 43: 1731-1737, 2004

2. Barua RS and Ambrose JA: Mechanisms of coronary thrombosis in cigarette smoke exposure. Arterioscler Thromb Vasc Biol 33: 1460-1467, 2013.

3. Shinton R and Beevers G: Meta-analysis of relation between cigarette smoking and stroke. BMJ 298: 789-794, 1989.

4. Price JF, Mowbray PI, Lee AJ, Rumley A, Lowe GD and Fowkes FG: Relationship between smoking and cardiovascular risk factors in the development of peripheral arterial disease and coronary artery disease. Euro Heart J 20: 344-353, 1999.

5. Barua RS, Sy F, Srikanth S, Huang G, Javed U, Buhari C, Margosan D and Ambrose JA: Effects of cigarette smoke exposure on clot dynamics and fibrin structure an ex vivo investigation. Arterioscler Thromb Vasc Biol 30: 75-79, 2010.

6. Swaminathan A, Amitkumar K, Ganapathy S and Ayyavoo S: Evaluation of the impact of cigarette smoking on platelet parameters. Natl J Physiol Pharm Pharmacol 5: 426-430, 2015.

7. Fusegawa Y, Goto S, Handa S, Kawada T and Ando Y: Platelet spontaneous aggregation in platelet-rich plasma is increased in habitual smokers. Thromb Res 93: 271-278, 1999.

8. Musallam KM, Rosendaal FR, Zaatari G, Soweid A, Hoballah JJ, Sfeir PM, Zeineldine S, Tamim HM, Richards T, Spahn DR, et al: Smoking and the risk of mortality and vascular and respiratory events in patients undergoing major surgery. JAMA Surg 148 755-762, 2013

9. Hawn MT, Houston TK, Campagna EJ, Graham LA, Singh J, Bishop $\mathrm{M}$ and Henderson WG: The attributable risk of smoking on surgical complications. Ann Surg 254: 914-920, 2011.

10. Wong J, Lam DP, Abrishami A, Chan MT and Chung F: Short-term preoperative smoking cessation and postoperative complications: A systematic review and meta-analysis. Can J Anaesth 59: 268-279, 2012.

11. Yousefzadeh A, Chung F, Wong DT, Warner DO and Wong J: Smoking cessation: The role of the anesthesiologist. Anesth Analg 122: 1311-1320, 2016.

12. Bombeli T and Spahn DR: Updates in perioperative coagulation: Physiology and management of thromboembolism and haemorrhage. Br J Anaesth 93: 275-287, 2004.

13. Chiang VL, Castleden WM and Leahy MF: Detection of reversible platelet aggregates in the blood of smokers and ex-smokers with peripheral vascular disease. Med J Aust 156: 601-603, 1992.

14. Morita H, Ikeda H, Haramaki N, Eguchi H and Imaizumi T: Only two-week smoking cessation improves platelet aggregability and intraplatelet redox imbalance of long-term smokers. J Am Coll Cardiol 4: 589-594, 2005.

15. Stegner D and Nieswandt B: Platelet receptor signaling in thrombus formation. J Mol Med (Berl) 89: 109-121, 2011

16. André P, Nannizzi-Alaimo L, Prasad SK and Phillips DR Platelet-derived CD40L: The switch-hitting player of cardiovascular disease. Circulation 106: 896-899, 2002.

17. Doi T, Adachi S, Matsushima-Nishiwaki R, Kato H, Enomoto Y, Minamitani C, Otsuka T, Tokuda H, Akamatsu S, Iwama T, et al: Antithrombin III suppresses ADP-induced platelet granule secretion: Inhibition of HSP27 phosphorylation. Arch Biochem Biophys 489: 62-67, 2009.

18. Enomoto Y, Adachi S, Matsushima-Nishiwaki R, Niwa M, Tokuda H, Akamatsu S, Doi T, Kato H, Yoshimura S, Ogura S, et al: AlphaB-crystallin extracellularly suppresses ADP-induced granule secretion from human platelets. FEBS Lett 583: 2464-2468, 2009.

19. Hanai Y, Adachi S, Yasuda I, Takai S, Matsushima-Nishiwaki R, Kato H, Enomoto Y, Akamatsu S, Sakakibara S, Ogura S, et al: Collagen-induced p38 MAP kinase activation is a biomarker of platelet hyper-aggregation in patients with diabetes mellitus. Life Sci 85: 386-394, 2009 .

20. Kato H, Adachi S, Doi T, Matsushima-Nishiwaki R, MinamitaniC, Akamatsu S, Enomoto Y, Tokuda H, Otsuka T, Iwama T, et al: Mechanism of collagen-induced release of 5-HT, PDGF-AB and sCD40L from human platelets: Role of HSP27 phosphorylation via p44/p42 MAPK. Thromb Res 126: 39-43, 2010.
21. Tohgi H, Takahashi H, Watanabe K, Kuki H and Shirasawa Y: Development of large platelet aggregates from small aggregates as determined by laser-light scattering: Effects of aggregant concentration and antiplatelet medication. Thromb Haemost 75: 838-843, 1996.

22. Tokuda H, Kato K, Kasahara S, Matsushima-Nishiwaki R, Mizuno T, Sakakibara S and Kozawa O: Significant correlation between the acceleration of platelet aggregation and phosphorylation of HSP27 at Ser-78 in diabetic patients. Int J Mol Med 30: 1387-1395, 2012

23. Kato $\mathrm{K}$, Ito $\mathrm{H}$, Hasegawa $\mathrm{K}$, Inaguma $\mathrm{Y}$, Kozawa $\mathrm{O}$ and Asano T: Modulation of the stress-induced synthesis of hsp27 and $\alpha \mathrm{B}$-crystallin by cyclic AMP in C6 rat glioma cells. J Neurochem 66: 946-950, 1996.

24. Laemmli UK: Cleavage of structural proteins during assembly of the head of bacteriophage T4. Nature 227: 680-685, 1970.

25. Rendu F and Brohard-Bohn B: The platelet release reaction: Granules' constituents, secretion and functions. Platelets 12: 263-271, 2001.

26. Ichiki $\mathrm{K}$, Ikeda $\mathrm{H}$, Haramaki $\mathrm{N}$, Ueno $\mathrm{T}$ and Imaizumi $\mathrm{T}$ : Long-term smoking impairs platelet-derived nitric oxide release. Circulation 94: 3109-3114, 1996.

27. Haramaki N, Ikeda H, Takajo Y, Katoh A, Kanaya S, Shintani S, Haramaki R, Murohara T and Imaizumi T: Long-term smoking causes nitroglycerin resistance in platelets by depletion of intraplatelet glutathione. Arterioscler Thromb Vasc Biol 21: 1852-1856, 2001.

28. Varol E, Icli A, Kocyigit S, Erdogan D, Ozaydin M and Dogan A: Effect of smoking cessation on mean platelet volume. Clin Appl Thromb Hemost 19: 315-319, 2013.

29. Takajo Y, Ikeda H, Haramaki N, Murohara T and Imaizumi T: Augmented oxidative stress of platelets in chronic smokers. Mechanisms of impaired platelet-derived nitric oxide bioactivity and augmented platelet aggregability. J Am Coll Cardiol 38: 1320-1327, 2001

30. Caponnetto P, Russo C, Di Maria A, Morjaria JB, Barton S, Guarino F, Basile E, Proiti M, Bertino G, Cacciola RR and Polosa R: Circulating endothelial-coagulative activation markers after smoking cessation: A 12-month observational study. Eur J Clin Invest 41: 616-626, 2011.

31. Grønkjær M, Eliasen M, Skov-Ettrup LS, Tolstrup JS, Christiansen AH,MikkelsenSS,BeckerU and Flensborg-Madsen T: Preoperative smoking status and postoperative complications: A systematic review and meta-analysis. Ann Surg 259: 52-71, 2014.

32. Al-Sarraf N, Thalib L, Hughes A, Tolan M, Youn V and McGovern E: Effect of smoking on short-term outcome of patients undergoing coronary artery bypass surgery. Ann Thorac Surg 86: 517-523, 2008.

33. Nakagawa M, Tanaka H, Tsukuma $\mathrm{H}$ and Kishi Y: Relationship between the duration of the preoperative smoke-free period and the incidence of postoperative pulmonary complications after pulmonary surgery. Chest 120: 705-710, 2001.

34. Turan A, Mascha EJ, Roberman D, Turner PL, You JY, Kurz A, Sessler DI and Saager L: Smoking and perioperative outcomes. Anesthesiology 114: 837-846, 2011.

35. Møller AM, Villebro N, Pedersen T and Tønnesen H: Effect of preoperative smoking intervention on postoperative complications: A randomised clinical trial. Lancet 359: 114-117, 2002.

36. Myers K, Hajek P, Hinds C and McRobbie H: Stopping smoking shortly before surgery and postoperative complications: A systematic review and meta-analysis. Arch Intern Med 171: 983-989, 2011.

37. Mason DP, Subramanian S, Nowicki ER, Grab DJ, Murthy SC, Rice TW and Blackstone EH: Impact of smoking cessation before resection of lung cancer: A Society of Thoracic Surgeons General Thoracic Surgery Database study. Ann Thorac Surg 88: 362-371, 2009.

This work is licensed under a Creative Commons Attribution-NonCommercial-NoDerivatives 4.0 International (CC BY-NC-ND 4.0) License. 